\title{
Neural Markers of Inhibition in Human Memory Retrieval
}

\author{
Maria Wimber, ${ }^{1,2}$ Karl-Heinz Bäuml, ${ }^{2}$ Zara Bergström, ${ }^{1}$ Gerasimos Markopoulos, ${ }^{1}$ Hans-Jochen Heinze, ${ }^{1}$ and \\ Alan Richardson-Klavehn ${ }^{1}$ \\ ${ }^{1}$ Department of Neurology, Otto von Guericke University, 39120 Magdeburg, Germany, and ${ }^{2}$ Department of Experimental Psychology, University of \\ Regensburg, Germany
}

Retrieving particular information from memory facilitates the later retrieval of that information, but also impairs the later retrieval of related, interfering information. It has been theorized that this retrieval-induced forgetting reflects inhibition of interfering memory representations. We used event-related fMRI to investigate the functional neuroanatomy of this impaired retrieval, at the time the impairment is observed. Neural activity differences between impaired and facilitated information occurred in left ventrolateral prefrontal cortex (VLPFC, BA 45 and 47), precuneus (BA 7), and right inferior parietal lobule (IPL, BA 40). Activity in left anterior VLPFC (BA 47) and left posterior temporal cortex (BA 22), regions implicated in the controlled retrieval of weak semantic memory representations, predicted the degree of retrieval-induced forgetting. In contrast, activity in precuneus and right IPL predicted the degree of retrievalinduced facilitation. Our findings demonstrate that impairment of interfering memories and facilitation of practiced memories involve distinct neural processes, and suggest that the impairment reflects inhibition that weakens interfering memory representations.

Key words: fMRI; memory; inhibition; interference; cognitive control; VLPFC

\section{Introduction}

The brain's ability to store vast quantities of information creates the problem of interference from currently irrelevant information during memory retrieval (Anderson and Neely, 1996; Levy and Anderson, 2002; Richardson-Klavehn and Bjork, 2002; Bäuml, 2008). Here we present evidence concerning the functional neuroanatomy of processes that attenuate such interference to permit goal-directed remembering.

Behavioral research demonstrates that selective retrieval of target information engages control processes that impair later retrieval of interfering memories (Anderson, 2003). In the retrieval practice paradigm, participants practice retrieval of a subset of previously studied items. In a final memory test, all studied items are to be remembered. Compared with a control condition without retrieval practice, practiced items show enhanced memory, but related but nonpracticed items show impaired memory, a finding called retrieval-induced forgetting (RIF).

It has been theorized that RIF reflects reduced availability of the nonpracticed information via inhibition (Anderson, 2003; Bäuml, 2008). Consistent with this hypothesis, behavioral evidence shows that RIF does not depend on how one attempts to access the impaired information. It occurs in direct memory tests like item recognition (e.g., Gómez-Ariza et al., 2005; Hicks and Starns, 2004; Spitzer and Bäuml, 2007) and cued recall (e.g.,

Received Sept. 5, 2008; accepted 0ct. 27, 2008.

The research was supported by the German Science Foundation (Deutsche Forschungsgemeinschaft) and the Federal State of Sachsen-Anhalt, Germany. We thank Kerstin Möhring and Ilona Wiedenhöft for assistance with scanning, and two anonymous reviewers for their helpful comments on a previous version of this article.

Correspondence should be addressed to Maria Wimber, Department of Neurology, Zenit 1 Building, Faculty of Medicine, Otto von Guericke University, Leipziger Strasse 44, 39120 Magdeburg, Germany. E-mail: wimber@med.ovgu.de.

DOI:10.1523/JNEUROSCI.1916-08.2008

Copyright $\odot 2008$ Society for Neuroscience $\quad$ 0270-6474/08/2813419-09\$15.00/0
Anderson et al., 1994; Bäuml et al., 2005), both with previously studied and novel cues (e.g., Anderson and Bell, 2001; Anderson and Spellman, 1995; Levy et al., 2007), and in indirect memory tests (e.g., Levy et al., 2007; Veling and Van Knippenberg, 2004).

There is, however, sparse evidence concerning the neural substrates of RIF, and most extant research has focused on brain activity during retrieval practice. Hemodynamic activations in anterior cingulate and lateral prefrontal cortex (Kuhl et al., 2007; Wimber et al., 2008), and a frontal positive slow-shift in eventrelated potentials (Johansson et al., 2007), predict greater later RIF, providing clues as to the substrates of this impairment at the time it is created. Only very recently has evidence emerged concerning the neural substrates of RIF at the later time when the impairment is observed, and this evidence does not directly address the inhibitory account of RIF (Kuhl et al., 2008).

We therefore optimized the retrieval practice paradigm for event-related fMRI during the final memory test, with the main goal of testing the inhibitory account of RIF against accounts that ascribe RIF to blocking of the nonpracticed information by the practiced information during the final memory test (e.g., Williams and Zacks, 2001). An influential neural model of controlled memory retrieval postulates that left anterior ventrolateral prefrontal cortex (VLPFC) subserves the controlled retrieval of weak memories, whereas mid-VLPFC subserves the selection of a memory among competing memories (Badre and Wagner, 2007). Based on this model, if the impaired retrieval of nonpracticed information reflects reduced memory availability via inhibition, this reduction should increase control demands, thus activating left anterior VLPFC. Moreover, the neural substrates of retrieval-induced impairment and enhancement should be dissociable. In contrast, if the impairment reflects blocking via competition from practiced information, retrieval of impaired mem- 

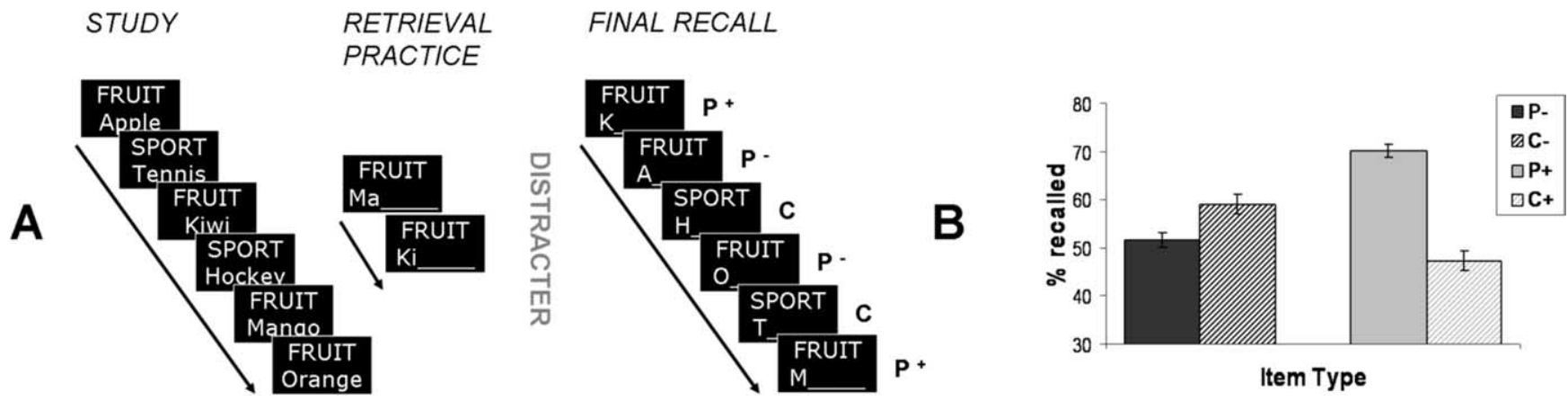

Figure 1. A, Schematic figure showing the behavioral procedure used in the present fMRI study. Each scanning session consisted of a study phase, during which exemplars from several categories were to be studied; a retrieval practice phase, during which half of the exemplars from two thirds of the categories were to be retrieved; a digit ordering distracter task; and a final recall phase, during which all initially studied exemplars were to be recalled. In the final recall, this procedure produced three different types of items: $\mathrm{P}^{+}$(retrieval practiced exemplars), $\mathrm{P}^{-}$(nonpracticed exemplars from practiced categories), and ( (control items from categories that did not participate at all in the retrieval practice phase). B, Costs and benefits of selective retrieval practice. Bars show mean percentage final cued recall performance (with SEMs) for the different item types. $\mathrm{P}^{-}$items showed $7.4 \%$ retrieval-induced forgetting compared with control items $\left(\mathrm{C}^{-}\right)$matched on normative strength; $\mathrm{P}^{+}$items showed $22.8 \%$ retrieval-induced enhancement compared with control items $\left(\mathrm{C}^{+}\right)$matched on normative strength.

ories should be associated with activation increases in mid-VLPFC.

\section{Materials and Methods}

Participants. Twenty-three right-handed native German speakers (10 male, mean age 23.5 years, $\mathrm{SD}=2.4$ ) were recruited at the University of Magdeburg for paid participation. They had no known history of neurological or psychiatric disease, had normal or corrected-to-normal vision, and gave their written informed consent. The experiment was conducted in accordance with the guidelines of the Ethics Commission of the University of Magdeburg Faculty of Medicine.

Behavioral procedure. The materials were 288 German nouns from 36 semantic categories ( 8 nouns per category), drawn from published norms (Battig and Montague, 1969; Scheithe and Bäuml, 1995). Within each category, all 8 items had unique first letters, and were divided into 4 normatively stronger and 4 normatively weaker exemplars of the category.

The experiment consisted of six separate scanning sessions, each comprising a study phase, a retrieval practice phase, a distracter phase, and a final recall test (Fig. $1 A$ ). In each study phase, participants were presented 48 items from 6 categories (e.g., SPORT-Volleyball; VEGETABLE-Zucchini; 8 items per category) in a pseudorandom order. Each study trial began with the presentation of a word-pair (category label plus category member) for $1200 \mathrm{~ms}$, followed by a fixation cross (+) for 1300 $\mathrm{ms}$, a question mark for $1200 \mathrm{~ms}$, and another fixation cross (+) for 800 ms. Upon presentation of the question mark, participants were instructed to respond with a yes/no button press whether the item was personally familiar to them or not. Eleven of the participants responded with the right hand, and the rest with the left hand. Participants always used their index finger for "yes" responses, and their middle finger for "no" responses.

In each retrieval practice phase, participants were asked to covertly complete unique word stems (2-4 initial letters, depending on word length) that corresponded to previously studied words (e.g., SPORTVoll___ in a random order. Each retrieval practice trial consisted of the presentation of the word stem for $1000 \mathrm{~ms}$, a fixation cross for 1300 ms, a question mark for $1200 \mathrm{~ms}$, and a fixation cross for $1000 \mathrm{~ms}$. Participants were instructed to covertly complete the word stems only with items from the immediately prior study list, and, upon presentation of the question mark, to indicate via button press if they could correctly remember the corresponding word. "Yes" responses were given with the index finger, "no" responses with the middle finger. Importantly, retrieval practice occurred for only half of the items out of two thirds of the studied categories in each scanning session. With this procedure, the 48 studied items could-after retrieval practice- be divided into three classes of items: 16 practiced items (henceforth called $\mathrm{P}^{+}$items; e.g., SPORT-Volleyball), 16 nonpracticed items out of practiced categories (henceforth called $\mathrm{P}^{-}$items; e.g., SPORT-Tennis), and 16 nonpracticed control items out of completely nonpracticed categories (henceforth called C items; e.g., VEGETABLE-Zucchini). In the behavioral literature, these conditions are typically labeled $\mathrm{Rp}^{+}, \mathrm{Rp}^{-}$, and $\mathrm{Nrp}$, respectively (see Anderson et al., 1994); here we adopt more straightforward labels for clarity.

In each category, the four exemplars with the lowest normative frequency as free associates to the category label served as $\mathrm{P}^{+}$items, and the four exemplars with the highest normative frequency served as $\mathrm{P}^{-}$items. This assignment was adopted to maximize the strength of the behavioral RIF effect, because previous behavioral work has shown that strong exemplars are more likely to elicit interference during retrieval practice, and are therefore more likely to be impaired by retrieval practice of the weak exemplars (Anderson et al., 1994; Bäuml, 1998). Because different recall rates are to be expected for higher and lower frequency exemplars, the control items were also separated into lower $\left(\mathrm{C}^{+}\right)$and higher $\left(\mathrm{C}^{-}\right)$ frequency exemplars. To control for possible effects of material, the lower frequency exemplars served both as $\mathrm{P}^{+}$and $\mathrm{C}^{+}$items, and the higher frequency exemplars served both as $\mathrm{P}^{-}$and $\mathrm{C}^{-}$items, across participants.

To exclude short-term memory effects, the retrieval practice phase was followed by a distracter task for $30 \mathrm{~s}$, in which participants had to indicate via button press if the first and the last out of 5 digits on the screen were arranged in an ascending manner or not. The index finger always corresponded to a "yes" answer, and the middle finger to a "no" answer.

During the final cued recall phases, all of the previously studied words had to be recalled. Participants were provided with category names together with the first letter of a study item (e.g., SPORT-T___), and were asked to overtly respond with the corresponding word, or to answer "next" whenever they did not know the correct answer. Each test trial began with the presentation of the category plus first letter cue for 1000 $\mathrm{ms}$, followed by a fixation cross for $1000 \mathrm{~ms}$, three exclamation marks for $1500 \mathrm{~ms}$, and another fixation cross for $1000 \mathrm{~ms}$. Items in the final recall phase of the experiment were arranged in pseudorandom order, such that the three different item types $\left(\mathrm{P}^{+}, \mathrm{P}^{-}\right.$, and $\left.\mathrm{C}\right)$ appeared equally often in each quarter of each test list. The oral recall responses were not to be given before presentation of the exclamation marks, meaning that they were always delayed until a $1500 \mathrm{~ms}$ window occurred $2000 \mathrm{~ms}$ after the presentation of each test cue. This was done to rule out any confound of speech onset latency with the effects of interest during item retrieval. Useable reaction time measurements were therefore not obtainable with this procedure. The oral responses were digitally recorded via a microphone fixed to the head coil for later behavioral scoring into correctly remembered and forgotten categories. An item was scored as remembered only if it exactly matched the target word on the study list.

The scanner was run continuously during each session consisting of study phase, retrieval practice phase, distracter phase, and final recall test. After the final recall test, scanning was interrupted for 1 or 2 min to allow participants a short break before the beginning of a new session. In the 
retrieval practice phases and the final recall tests, trials were interspersed with null events in pseudorandom order, to allow better modeling of the event-related hemodynamic response functions. Null trials were similar to "real" trials, except that in the beginning of a null trial, a prolonged fixation cross $(+)$ was presented instead of an item. In these null trials, participants always pressed the left response button (retrieval practice phases), or responded with "next" (final recall phases), upon presentation of the question mark or exclamation marks, respectively, to control for motor and speech activity. The study phases did not include null trials.

For the analysis of the behavioral data, two-tailed $t$ tests with a statistical threshold of $p<0.05$ were performed. Retrieval-induced forgetting was calculated as the difference between $\mathrm{P}^{-}$items and the matched $\mathrm{C}^{-}$ control items for each participant. Likewise, enhancement through retrieval practice was calculated as the difference between $\mathrm{P}^{+}$items and the matched $\mathrm{C}^{+}$control items for each participant. Analyses of brain-behavior correlations (see below) used these individual-participant values.

fMRI data acquisition and analysis. Brain activity was measured using a GE Medical Systems Signa 1.5 T MRI research scanner belonging to the University of Magdeburg Faculty of Medicine. Functional images were acquired using an interleaved (bottom to top) echo-planar imaging sequence (repetition time $=2000 \mathrm{~ms}$, echo time $=35 \mathrm{~ms}$ ) sensitive to blood-oxygenation-level-dependent (BOLD) contrast. In each of the six sessions (each session consisting of study phase, retrieval practice phase, distracter phase, and final recall phase), 342 whole-brain volumes were acquired, with 72 volumes in each retrieval practice phase, and 144 volumes in each final recall phase. The first 3 volumes of each session were discarded to guarantee steady state tissue magnetization. Images consisted of 23 axial slices, with a slice thickness of $5 \mathrm{~mm}$ plus $1 \mathrm{~mm}$ gap, and an in-plane resolution of $3.15 \times 3.15 \mathrm{~mm}$. High-resolution T1-weighted anatomical images for visualization purposes were already available from each participant. Head movement was restricted using pillows and foam inserts, and participants were instructed to move as little as possible, especially during the final recall phases, which involved overt speech.

Data preprocessing and statistical analysis were performed using statistical parametrical mapping software (SPM2, Wellcome Department of Cognitive Neurology, London, UK: www.fil.ion.ucl.ac.uk/spm/). Functional images were temporally and spatially realigned, coregistered to the anatomical images, normalized to an average T1 template in standard stereotactic MNI space (Montreal Neurological Institute, Montreal, Quebec, Canada: http://www2.bic.mni.mcgill.ca/), and finally smoothed with a Gaussian kernel of $8 \mathrm{~mm}$ at FWHM. Given the overt speech responses in the final recall phases of the experiment, the results of spatial realignment were carefully examined for excessive movement within runs. Mean within-run movement was $0.76 \mathrm{~mm}(\mathrm{SD}=0.21)$ translation, and 0.016 degrees $(\mathrm{SD}=0.008)$ rotation, and no participants needed to be excluded owing to excessive movement.

For first level (single participant) statistical analyses, event-related hemodynamic responses were modeled with delta stick functions at the onset of each event of interest, convolved with a first order canonical hemodynamic response function (Friston et al., 1994). The resulting time series of single voxels were then used to form covariates in a fixed effects general linear model. All trials were modeled as two separate events, one for the stimulus presentation, and one for the motor response (study and retrieval practice phases) or oral response (final recall phases). For the study and final recall phases, remembered and forgotten items were modeled separately, as were items of the type $\mathrm{P}^{+}$(practiced), $\mathrm{P}^{-}$ (nonpracticed competitor), $\mathrm{C}^{+}$(weak control), and $\mathrm{C}^{-}$(strong control). Analysis of the study and retrieval practice phases additionally included a regressor to capture the motor responses, and analysis of the final recall phases additionally included a regressor for speech events. In the latter case, modeling the speech events separately from the retrieval cue presentations followed a now widely accepted analysis procedure for fMRI experiments using oral cued recall (e.g., Schott et al., 2005). Session-specific effects, as well as the six rigid-body movement parameters determined from realignment, were included as separate covariates. Statistical parametric maps of linear contrasts were estimated, with lowfrequency signal components (cutoff $128 \mathrm{~s}$ ) treated as confounds. Planned comparisons at the first level included the contrasts between $\mathrm{P}^{-}$ and $\mathrm{C}$ items, between $\mathrm{P}^{+}$and $\mathrm{C}$ items, and between $\mathrm{P}^{-}$and $\mathrm{P}^{+}$items. These contrasts could only be estimated for remembered items, because the high recall performance for $\mathrm{P}^{+}$items did not leave enough statistical power for a comparison between forgotten items.

The first level contrast estimates were entered into a second level analysis, with participants treated as a random factor. Individual parameter estimates ( $\beta$ weights) for event-related responses during retrieval practice trials were tested with one-sample $t$ tests against the hypothesis of a zero $\beta$ value. Mean differences between estimates for event-related responses during the final recall test were tested with one-sample $t$ tests against the hypothesis of a zero difference. These contrasts were performed for differences between $\mathrm{P}^{-}$and $\mathrm{C}$ items, $\mathrm{P}^{+}$and $\mathrm{C}$ items, and $\mathrm{P}^{-}$ and $\mathrm{P}^{+}$items, with $\mathrm{C}$ items collapsed across $\mathrm{C}^{+}$and $\mathrm{C}^{-}$items. Later regions of interest (ROI) analyses separated the two types of $\mathrm{C}$ items. A simple regression model was used to assess linear relationships between behavioral indices and brain activation. For these brain-behavior correlations, behavioral indices of retrieval-induced forgetting (RIF in \%) and retrieval-induced enhancement (enhancement in \%), calculated for each participant as previously described, were used to predict differences in neural activity during the final recall of $\mathrm{P}^{-}$compared with $\mathrm{P}^{+}$items. Unless otherwise mentioned, differences and correlations were considered significant if they reached a threshold of $p<0.001$, uncorrected for multiple comparisons, and only if a cluster contained 10 or more adjacent voxels.

\section{Results}

Data presented here are pooled across the six sessions, each consisting of study phase, retrieval practice phase, distracter phase, and final cued recall test phase.

\section{Behavioral results}

During retrieval practice, participants indicated via button press that they were successful in completing a given word stem with an item from the study list in $64.1 \%$ of the trials. Although this success rate is based on a subjective measure, it was positively correlated, across participants, with later $\mathrm{P}^{+}$performance in the final recall tests $(r=0.69)$, and with average memory performance across all item types $(r=0.49)$ in the final recall tests.

The final cued recall results (Fig. $1 B$ ) revealed significantly greater recall of previously practiced $\mathrm{P}^{+}$items $(\mathrm{M}=70.1 \%)$ compared with the matching nonpracticed $\mathrm{C}^{+}$control items out of previously nonpracticed categories $(\mathrm{M}=47.3 \%)$, which corresponded to an average retrieval-induced enhancement of $22.8 \%(\mathrm{SE}=1.9 \%)$. More importantly, nonpracticed $\mathrm{P}^{-}$items out of previously practiced categories $(\mathrm{M}=52.6 \%)$ were recalled significantly worse than the matching nonpracticed $\mathrm{C}^{-}$control items out of previously nonpracticed categories $(\mathrm{M}=59.0 \%)$, which corresponded to an average of $7.4 \%$ retrieval-induced forgetting $(\mathrm{SE}=2.7 \%$ ). Thus, our behavioral results are in accordance with previous findings on retrieval-induced forgetting (Anderson, 2003): selective retrieval practice of only some items associated with a category cue (i.e., $\mathrm{P}^{+}$items) later led to the enhanced retrieval of those items, and to the impaired retrieval of nonpracticed but competing items that were bound to the same category cue (i.e., $\mathrm{P}^{-}$items), compared with matched control items from categories that were studied, but did not appear in the retrieval practice phase.

\section{fMRI results}

Although fMRI was measured during all phases of each session, we report here only the data collected during the retrieval practice phases and the subsequent final recall phases.

\section{Retrieval practice phase}

Event-related activity during retrieval practice trials showed significant hemodynamic response increases compared with fixa- 
tion baseline in prefrontal, occipital, and medial temporal lobes (Fig. 2; supplemental Table 1, available at www.jneurosci.org as supplemental material). Prefrontal activations included one cluster extending over left premotor and ventrolateral prefrontal areas (VLPFC, Brodmann area $6 / 44 / 45$ ), and one in the medial presupplementary motor area (preSMA, BA 6). Posterior activations extended bilaterally from late visual regions (BA 18/19) to the superior parietal cortex (BA 7). Moreover, both the left and right hippocampus showed increased signal relative to baseline. Areas showing hemodynamic response decreases relative to fixation baseline included the medial (BA 32 and BA 6) and lateral (BA 8) prefrontal cortices, the right supramarginal gyrus (BA 40), and right lateral temporal regions (BA 20/21).

\section{Final recall phase}

We first calculated the contrast between the impaired $\mathrm{P}^{-}$and the unimpaired control (C) items, because this comparison parallels the behavioral contrast for calculating retrieval-induced forgetting. From an imaging point of view, the contrast between $\mathrm{P}^{-}$and $\mathrm{C}$ items may be contaminated by the effects of differential category familiarity. More specifically, $\mathrm{P}^{-}$ items, although not explicitly practiced, come from categories that are presented several times during retrieval practice. Therefore, differences between $\mathrm{P}^{-}$and $\mathrm{C}$ items are very likely not genuinely related to impairment, but might simply be related to increased familiarity of practiced categories. If so, activations related to category priming should also be reflected in the contrast between $\mathrm{P}^{+}$and $\mathrm{C}$ items.

Significant hemodynamic increases for $\mathrm{P}^{-}$items were found bilaterally in the supramarginal gyrus (BA 40), bilaterally in the anterior cingulate (BA 32), and in the right superior frontal gyrus (BA 8). As shown in detail in supplemental Table 2 (available at www.jneurosci.org as supplemental material), these activations mostly overlapped with the results of the comparison between $\mathrm{P}^{+}$and $\mathrm{C}$ items, which also yielded hemodynamic increases in the left and right supramarginal gyrus (BA 40), the anterior cingulate (BA 32), and the right superior frontal gyrus (BA 8), but with one additional activation in the precuneus (BA 31) for $\mathrm{P}^{+}$ items (see Fig. 3A).

Hemodynamic response decreases during the final recall of $\mathrm{P}^{-}$compared with control items and $\mathrm{P}^{+}$compared with control items were more widespread, but also found mainly in overlapping regions (for a complete list of peak activations, see supplemental Table 3, available at www.jneurosci.org as supplemental material), with both comparisons including one large bilateral posterior cluster extending over late visual areas (BA 17/18) and the precuneus (BA 19), and two left prefrontal clusters, one extending medially from the supplementary motor area (BA 6) to the cingulate gyrus (BA 32), and the second one covering the area from the left lateral precentral gyrus (BA 6) to the inferior frontal gyrus (BA 45). Moreover, the bilateral inferior frontal area 47 emerged in both of the above contrasts, with the deactivations in this area being more left lateralized in $\mathrm{P}^{-}$compared with $\mathrm{C}$ items, and more right lateralized in $\mathrm{P}^{+}$compared with $\mathrm{C}$ items (for a graphical overview, see Fig. 3B).

\section{Impairment and enhancement during final recall}

The planned comparisons described above revealed the expected finding that $\mathrm{P}^{-}$and $\mathrm{P}^{+}$items, compared with control items, elicited overall very similar hemodynamic responses during final recall, which are likely related to increases in category familiarity through prior practice. Therefore, we tested for areas where $\mathrm{P}^{-}$ and $\mathrm{P}^{+}$items directly differed from each other (see Fig. 3, blue regions; Table 1). Importantly, the contrast between $\mathrm{P}^{-}$and $\mathrm{P}^{+}$ items is not contaminated by differential category familiarity, and may therefore be best suited to isolate activations specifically related to inhibition and enhancement. With this direct comparison, we found that $\mathrm{P}^{-}$recall elicited greater hemodynamic activity in two ventrolateral prefrontal regions, one in ventrolateral

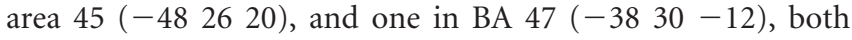
left-lateralized. The reverse comparison yielded the strongest hemodynamic decreases during $\mathrm{P}^{-}$recall compared with $\mathrm{P}^{+}$recall in the precuneus (BA 7), the supramarginal gyrus (BA 40), and in the orbital part of the medial frontal wall (BA 11).

Figure 4 shows parameter estimates extracted from BA 45 and BA 47, separately for $\mathrm{P}^{+}, \mathrm{P}^{-}, \mathrm{C}^{+}$, and $\mathrm{C}^{-}$items. In left anterior VLPFC (BA 47), we found a significant interaction between practice status ( $\mathrm{P}$ or $\mathrm{C}$ ) and a priori item strength (weak + or strong $-), F_{(1,22)}=22.82, p<0.001$, but no main effect of practice status $\left(F_{(1,22)}=2.48, p=0.13\right)$ or a priori strength $\left(F_{(1,22)}=0.23\right.$, $p=0.64)$. In contrast, left mid-VLPFC showed no significant practice by strength interaction $\left(F_{(1,22)}=3.05, p=0.09\right)$ and no significant main effect of practice $\left(F_{(1,22)}=1.02, p=0.32\right)$, but a significant main effect of a priori strength $\left(F_{(1,22)}=7.20, p<\right.$ $0.05)$. These findings support the view that BA 47 responds to semantic item strength (Badre et al., 2005), being more active during the retrieval of weak control items $\left(\mathrm{C}^{+}\right)$than during the retrieval of strong control items $\left(\mathrm{C}^{-}\right)$. However, strikingly, this difference reversed as a result of prior retrieval practice, with BA 47 showing more activation during the retrieval of the normatively strong but impaired $\mathrm{P}^{-}$items than during the retrieval of normatively weak but facilitated $\mathrm{P}^{+}$items. BA 45 did not respond differentially to normatively weak and strong control items. This observation is in line with previous findings showing that BA 45 is not sensitive to cue-target associative strength, but increases response when, for example, a target item must be selected from 

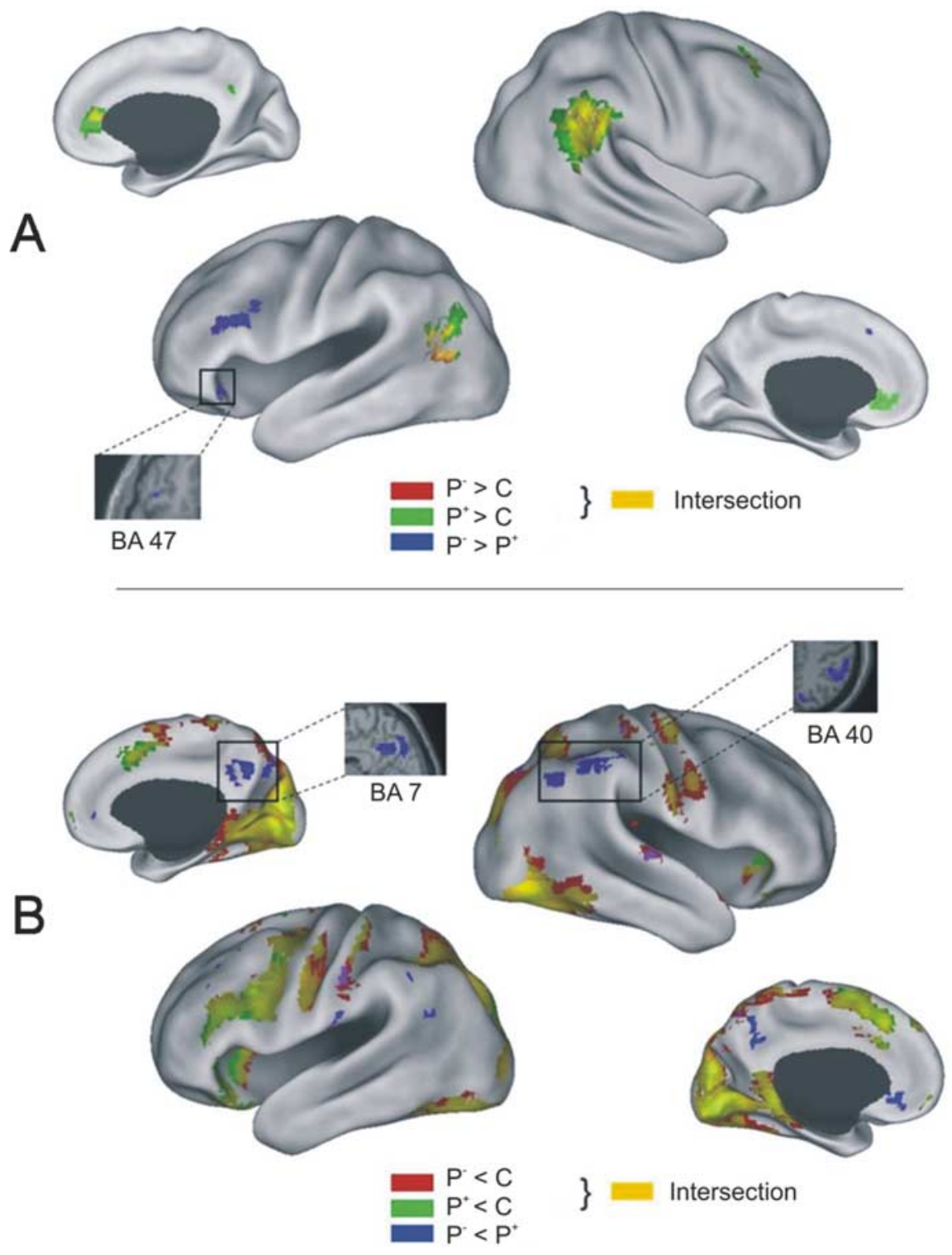

Figure 3. Positive $(\boldsymbol{A})$ and negative $(\boldsymbol{B})$ hemodynamic signal differences between item types during the final cued recall test, overlaid onto a flattened standard cortical surface in SPM2 space. Yellow coloring indicates areas where $\mathrm{P}^{-}$(red) and $\mathrm{P}^{+}$(green) items both significantly differed from $C$ control items, while blue coloring indicates areas where $\mathrm{P}^{-}$and $\mathrm{P}^{+}$items significantly differed in hemodynamic signal. Highlighted are regions where the contrast between $\mathrm{P}^{-}$and $\mathrm{P}^{+}$items significantly correlated with retrieval-induced forgetting or enhancement (see also Fig. 5).

memory in the context of interference from more compared with less competing items (Badre et al., 2005).

The neural differences between $\mathrm{P}^{-}$and $\mathrm{P}^{+}$retrieval cannot be attributed solely to the impairment of $\mathrm{P}^{-}$items, but might also occur due to the facilitated retrieval of $\mathrm{P}^{+}$items, or due to stronger repetition suppression for explicitly practiced $\mathrm{P}^{+}$items. To assess which areas showed impairment-specific effects during final recall, we examined activation differences between $\mathrm{P}^{-}$and $\mathrm{P}^{+}$items that varied with the degree to which the individual participants showed the behavioral retrieval-induced forgetting effect (see Table 2 and Fig. 5). The two areas showing the strongest correlation with retrieval-induced forgetting were located in the left superior temporal gyrus (BA 22, $r=0.74$ ), and in the left inferior frontal gyrus (BA 47, $r=0.69$ ). The activation difference between $\mathrm{P}^{-}$and $\mathrm{P}^{+}$items in these areas was not significantly correlated with retrieval-induced enhancement of the $\mathrm{P}^{+}$items $(r=-0.32$ in BA 47, $r=0.04$ in BA 22). Regions that correlated with the level of $\mathrm{P}^{+}$enhancement through retrieval practice were located in right medial and lateral parietal areas (see Table 2 and Fig. 5). Here, the strongest correlations were found in the right precuneus (BA 7, $r=0.74$ ), and in the right lateral inferior parietal lobe (BA 40, $r=0.56$ ). To assess if activation in these parietal regions predicted activation in the inferior frontal cortex, we used the difference between $\mathrm{P}^{-}$ and $\mathrm{P}^{+}$item retrieval in BA 40 and BA 7 as a regressor for predicting activation in a ROI including the left and right inferior frontal gyri. BA 40 showed a significant correlation with activation in one region in the right posterior IFG (BA 45, 5612 24, $r=0.64$ ), whereas BA 7 predicted activation in right anterior IFG (BA 47, 3018 $-16, r=0.59)$.

An additional ROI analysis was performed to test for across-participants correlations between retrieval-induced forgetting and BA 45 and BA 47, as defined by the contrast between $\mathrm{P}^{-}$and $\mathrm{P}^{+}$retrieval activation (see Figs. 3A, 4). Whereas activation in BA 47 was significantly correlated with RIF $(r=0.73, p<0.0001)$, the correlation between RIF and BA 45 activation did not reach significance $(r=0.31$, $p>0.1)$. Furthermore, the correlation between activity in BA 45 and RIF was significantly larger $\left(t_{(20)}=2.78, p=0.012\right.$, twotailed) than the correlation between activity in BA 45 and RIF, as calculated using Williams' $t$ test for the difference between two dependent correlations (Williams, 1959). This analysis provides further evidence that BA 47, but not BA 45 , is functionally related to retrieval-induced forgetting during the final recall of $\mathrm{P}^{-}$ items.

To test for functional coupling between posterior and prefrontal regions, we extracted contrast estimates from the BA 22 cluster that predicted forgetting, and from the BA 45 and BA 47 clusters that showed a significant difference between $\mathrm{P}^{-}$and $\mathrm{P}^{+}$recall. Across participants, activation in BA 22 was significantly correlated with activation in BA $47(r=0.73, p<0.0001)$, but not in BA $45(r=0.36$, $p=0.10)$. The results from the final recall phase of the experiment thus show that retrieval-induced forgetting is mainly related to activation in left inferior frontal regions. Notably, activity in anterior VLPFC (BA 47) showed a strong correlation with forgetting, and functionally coupled with activity in left temporal BA 22.

\section{Relationship between retrieval practice and final recall}

Finally, we performed an additional post hoc analysis regarding the relationship between prefrontal involvement during retrieval practice and the final recall test. Based on the above results of the comparison between $\mathrm{P}^{-}$and $\mathrm{P}^{+}$items, the brain-behavior correlations, and previous imaging work (Badre and Wagner, 2007), we hypothesized that if activation in left anterior VLPFC (BA 47) 
does indeed reflect the impaired state of $\mathrm{P}^{-}$items, there should be inferior frontal selection-related activations during retrieval practice that predict later BA 47 activation during final recall. We therefore extracted BA 47 activation during the final recall (ROI defined as the cluster activated during $\mathrm{P}^{-}>\mathrm{P}^{+}$retrieval), and used the resulting estimates as a regressor for eventrelated activation during retrieval practice, restricting the analysis to bilateral inferior prefrontal cortices. The two regions that showed a significant $(p<0.005$, uncorrected) correlation with later left anteriorVLPFC activation were located in left BA $47(-2424-16, t=4.71,42$ voxels; $r=$ $0.71)$, and in left BA 44/45 (-52 $924, t=$ $3.75,38$ voxels; $r=0.64)$, as shown in Figure 2. Note that this BA 47 focus was in a more orbital region of the inferior frontal gyrus than the one found in the $\mathrm{P}^{-}$versus $\mathrm{P}^{+}$contrast during final recall. These findings suggest that both anterior and midVLPFC play a crucial role during retrieval practice, and are related to later anterior VLPFC activation for impaired compared with practiced items during final recall.
Table 1. Peak locations showing significant hemodynamic differences in the direct comparison between $\mathrm{P}^{-}$and $P^{+}$items $(p<0.001$, uncorrected for multiple comparisons, extent threshold 10 voxels) during the final recall test (see also Fig. 3)

\begin{tabular}{lrrrrrr}
\hline Anatomical label & \multicolumn{1}{c}{ X } & \multicolumn{1}{c}{ B } & BA & $t$ & Size \\
\hline $\mathrm{P}^{-}>\mathrm{P}^{+}$ & & & & & & \\
L inferior frontal G. & -48 & 26 & 20 & 45 & 4.73 & 49 \\
& -36 & 16 & 24 & 45 & 4.01 & \\
L inferior frontal G. & -38 & 30 & -12 & 47 & 4.12 & 14 \\
$\mathrm{P}^{+}>\mathrm{P}^{-}$ & & & & & & \\
B precuneus & 2 & -54 & 36 & 7 & 6.16 & 176 \\
& 8 & -62 & 36 & 7 & 4.88 & \\
& -10 & -56 & 36 & 7 & 4.08 & \\
R inferior parietal lobule & 40 & -48 & 44 & 40 & 5.24 & 167 \\
& 46 & -54 & 40 & 40 & 4.77 & \\
& 46 & -66 & 40 & 39 & 4.14 & \\
L precuneus & -10 & -64 & 48 & 7 & 5.09 & 42 \\
L cerebellum & -8 & -46 & -20 & $N A$ & 5.06 & 20 \\
R precuneus/superior parietal lobe & 10 & -74 & 32 & 7 & 5.06 & 105 \\
& 16 & -66 & 56 & 7 & 4.96 & \\
L medial frontal G. & 10 & -70 & 52 & 7 & 3.93 & \\
& -6 & 32 & -12 & 11 & 4.70 & 155 \\
L postcentral G. & -2 & 44 & -8 & 10 & 4.12 & \\
L supramarginal/angular G. & -60 & -14 & 24 & $3 / 4$ & 4.62 & 23 \\
L basal ganglia (putamen) & -52 & -54 & 28 & $39 / 40$ & 4.51 & 20 \\
R superior temporal G. & -26 & 0 & 4 & & 4.49 & 29 \\
L precentral G. & 64 & -22 & 0 & 22 & 4.38 & 15 \\
R anterior cingulate & -54 & -16 & 44 & 4 & 4.12 & 12 \\
\hline
\end{tabular}

$B A$, Approximate Brodmann Area; L, left hemisphere; $R$, right hemisphere; $B$, bilateral; size, number of voxels in a cluster.

\section{Discussion}

The present fMRI study investigated the functional neuroanatomy of the detrimental and facilitatory effects that prior retrieval practice can have on the recall of long-term memories, at the very time the impairment and enhancement are observed. We were able to demonstrate that retrieval-induced forgetting and enhancement have distinct neural substrates during final recall. Moreover, the results suggest that the impairment is the product of an inhibitory process that weakens interfering memory representations.

Prior imaging investigations have examined the neural basis of retrieval-induced forgetting during retrieval practice (Kuhl et al., 2007; Wimber et al., 2008). In contrast, our primary goal was to identify the neural markers during final recall that might indicate the impaired state of $\mathrm{P}^{-}$items. The comparison of $\mathrm{P}^{-}$and $\mathrm{P}^{+}$with control items suggests that there was strong repetition priming (e.g., Henson and Rugg, 2003; Schacter et al., 2007) during the final recall of both item types, likely due to repeated category presentation during practice. To search for specific neural correlates of enhancement and impairment, a direct comparison between $\mathrm{P}^{-}$and $\mathrm{P}^{+}$items revealed that there were indeed reliable activation differences. Final recall of $\mathrm{P}^{-}$items, compared with $\mathrm{P}^{+}$items, was associated with more activation in two left ventrolateral prefrontal areas (BA 45 and BA 47), and with less activation in medial (BA 7) and lateral (BA 40) parietal areas. Moreover, activation in anterior left VLPFC and in posterior lateral temporal cortex was highly predictive of the degree to which participants showed retrieval-induced forgetting. In contrast, activation in the right medial and lateral parietal cortex was highly predictive of the degree to which participants showed retrievalinduced enhancement. Thus, the brain-behavior correlations show dissociable brain substrates of impairment and enhancement during final recall, with prefrontal differences reflecting the impairment of the nonpracticed $\mathrm{P}^{-}$items, and parietal differences reflecting the enhancement of the practiced $\mathrm{P}^{+}$items.

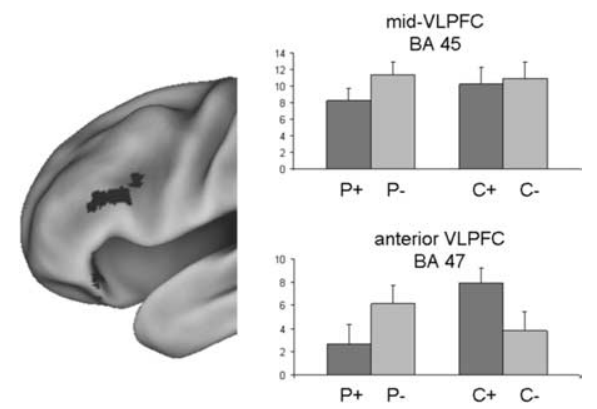

Figure 4. Mid-VLPFC and anterior VLPFC responses ( $y$-axis shows eigenvariates extracted from the ROIs) during the final recall of the different item types. Mid-VLPFC (BA 45, - 482620) showed no significant activity difference during recall of normatively strong $\left(\mathrm{C}^{-}\right)$and normatively weak $\left(\mathrm{C}^{+}\right)$control items, but increased activity during recall of $\mathrm{P}^{-}$compared with $\mathrm{P}^{+}$ items. In contrast, anterior VLPFC (BA 47, - 3830 - 12) showed increased activity during recall of normatively weak $\left(\mathrm{C}^{+}\right)$compared with normatively strong $\left(\mathrm{C}^{-}\right)$control items, but increased activity during recall of normatively strong but impaired $\mathrm{P}^{-}$items compared with normatively weak but enhanced $P^{+}$items, suggesting that the memory representations of the normatively strong $\mathrm{P}^{-}$items were weakened by retrieval practice.

Previous evidence indicates that different subregions of the VLPFC subserve different processes during long-term memory retrieval. The anterior VLPFC is assumed to support controlled retrieval by activating semantic knowledge in the lateral temporal cortex, whereas the mid-VLPFC has been linked to post-retrieval selection processes, which are more generally required whenever relevant information has to be selected against competing information (Badre et al., 2005; Badre and Wagner, 2007). For example, anterior VLPFC selectively activates when weak associates to a given cue word are to be retrieved (Badre et al., 2005), and when the interstimulus interval is long enough to allow controlled retrieval (Gold et al., 2006). In contrast, mid-VLPFC selectively activates when irrelevant, competing primes for a target word are presented (Gold et al., 2006), or when the number of competing 


\begin{tabular}{|c|c|c|c|c|c|c|}
\hline Anatomical label & $x$ & $y$ & $Z$ & $\mathrm{BA}$ & $t$ & Size \\
\hline \multicolumn{7}{|l|}{ Correlation with forgetting } \\
\hline \multirow[t]{3}{*}{ L superior/middle temporal G. } & -50 & -58 & 12 & 22 & 5.81 & 51 \\
\hline & -58 & -50 & 8 & 22 & 4.2 & \\
\hline & -52 & -44 & 4 & 22 & 3.74 & \\
\hline \multirow[t]{3}{*}{ L inferior frontal G. } & -42 & 26 & -4 & 47 & 4.79 & 129 \\
\hline & -44 & 18 & 4 & 45 & 4.49 & \\
\hline & -42 & 40 & -8 & 47 & 3.98 & \\
\hline \multirow[t]{2}{*}{ L thalamus (ventral posterior lateral nucleus) } & -22 & -20 & 8 & NA & 4.62 & 26 \\
\hline & -14 & -16 & 8 & NA & 4.05 & \\
\hline R inferior frontal G. & 48 & 32 & -8 & 47 & 4.5 & 40 \\
\hline \multicolumn{7}{|l|}{ Correlation with enhancement } \\
\hline R precuneus & 22 & -72 & 36 & 7 & 5.66 & 23 \\
\hline R inferior parietal lobule & 62 & -36 & 40 & 40 & 5.3 & 46 \\
\hline R posterior cingulate $\mathrm{G}$. & 16 & 48 & 28 & 31 & 4.5 & 15 \\
\hline R precuneus & 12 & 56 & 36 & 7 & 4.07 & 12 \\
\hline
\end{tabular}

$\mathrm{BA}$, Approximate Brodmann area; $\mathrm{L}$, left hemisphere; $R$, right hemisphere; size, number of voxels in a cluster.

targets is increased (Badre et al., 2005). Moreover, anterior VLPFC, but not mid-VLPFC, functionally couples with lateral temporal regions thought to store semantic representations (Badre et al., 2005; Gold et al., 2006). Together, these findings have been taken as evidence for a two-process account of left VLPFC function (Badre and Wagner, 2007), with the anterior portion subserving controlled retrieval, and the dorsocaudal portion subserving retrieval selection in the face of competition.

Provided the two-process account is correct, our finding that activity in area 47 and the lateral temporal cortex predicts retrieval-induced forgetting is especially relevant with respect to the two alternative views of how retrieval practice leads to later forgetting of nonpracticed information. According to the inhibitory account, inhibitory processes act on the nonpracticed $\mathrm{P}^{-}$ items during retrieval practice of $\mathrm{P}^{+}$items, weakening the memory representations of $\mathrm{P}^{-}$items (e.g., Anderson and Spellman, 1995; Spitzer and Bäuml, 2007). According to blocking theory, $\mathrm{P}^{+}$items are strengthened during retrieval practice and subsequently block access to the nonpracticed $\mathrm{P}^{-}$items during final recall, without affecting the nonpracticed items' memory representations (e.g., Williams and Zacks, 2001; Rundus, 1973). Regarding VLPFC involvement, the inhibitory account predicts that the final recall of $\mathrm{P}^{-}$items requires more controlled retrieval, presumably supported by anterior VLPFC and lateral temporal areas (Badre and Wagner, 2007), because their weakened representations are temporarily less available. In contrast, blocking accounts predict that the recall of $\mathrm{P}^{-}$items makes higher demands on selection processes, presumably supported by midVLPFC, because practiced $\mathrm{P}^{+}$items block access to related $\mathrm{P}^{-}$ items.

We found that both anterior and mid-VLPFC showed stronger activation during $\mathrm{P}^{-}$compared with $\mathrm{P}^{+}$recall, suggesting that both controlled retrieval and competition play a role during the retrieval of impaired items. However, this activation difference was related to the degree of retrieval-induced forgetting in the anterior VLPFC (see Table 2), but not in the mid-VLPFC, as confirmed by a ROI analysis. Moreover, we found a strong positive correlation with retrieval-induced forgetting in the lateral temporal cortex, and in line with the two-process view (Badre and Wagner, 2007), this area functionally coupled with the anterior VLPFC, but not with mid-VLPFC. The highest activation in BA 47 occurred during the recall of impaired $\mathrm{P}^{-}$and normatively weak $\mathrm{C}^{+}$items (see Fig. 4). This pattern suggests that activity in
BA 47 is primarily sensitive to the momentary availability of a memory trace. Finally, anterior VLPFC activation during $\mathrm{P}^{-}$final recall was predicted by both anterior and mid-VLPFC activation during retrieval practice (see Fig. 2), suggesting that both controlled retrieval and selection processes play a crucial role for determining the later availability of memories that compete during retrieval practice.

We interpret these findings as reflecting increased demands on controlled retrieval during the final recall of $\mathrm{P}^{-}$items, caused by an inhibitory process during retrieval practice that reduces their later memory availability. Strengthening this conclusion, activity in both anterior VLPFC and lateral temporal cortex did not correlate with the degree of enhancement of the $\mathrm{P}^{+}$items, instead showing a trend in the opposite direction. Regions correlated with the degree of retrieval-induced enhancement were located more posteriorly, in the medial and lateral parietal cortex, possibly reflecting the increased accessibility of practiced items (Wagner et al., 2005). A similar dissociation between the neural substrates of impairment and enhancement has recently been reported in an electrophysiological study (Spitzer et al., 2008). In this study, impaired recognition of $\mathrm{P}^{-}$items was associated with early frontal ERP and theta power effects, whereas enhanced recognition of $\mathrm{P}^{+}$items was associated with late parietal ERP and alpha power effects, providing further evidence for the inhibitory view, according to which impairment and enhancement are mediated by distinct processes.

The hypothesis that retrieval-induced forgetting results from an inhibition process that renders interfering items at least temporarily less available has previously been based on behavioral modeling work (e.g., Spitzer and Bäuml, 2007), and on behavioral results indicating that retrieval-induced forgetting occurs independently of the way participants try to access the impaired items, including in incidental tests (e.g., Veling and Van Knippenberg, 2004) and tests providing novel cues (e.g., Anderson et al., 2000; Anderson and Spellman, 1995; Johnson and Anderson, 2004). These findings argue against a blocking interpretation of retrieval-induced forgetting, because they suggest that the memory representation of the impaired information has itself been rendered less available. Here we found neural evidence for such inhibitory effects.

This evidence is of particular interest in view of recent findings of Kuhl et al. (2008), who also studied retrieval-induced forgetting during final recall. They found that anterior cingulate, right VLPFC and left mid-VLPFC increased activity during $\mathrm{P}^{-}$compared with $\mathrm{P}^{+}$retrieval, and that activity in anterior cingulate and right VLPFC correlated with $\mathrm{P}^{-}$forgetting. The involvement of left mid-VLPFC in retrieving impaired memories, and the correlation of right VLPFC with retrieval-induced impairment, are consistent with the present data. Moreover, our finding of a correlation between parietal and right VLPFC activity for the $\mathrm{P}^{-}$ versus $\mathrm{P}^{+}$contrast fits with these authors' argument that right VLPFC activation reflects increased demands on attentional selection during final recall of $\mathrm{P}^{-}$items due to increased accessibility of $\mathrm{P}^{+}$items. However, they did not report activation in left anterior VLPFC, which in the present experiment showed the 
A

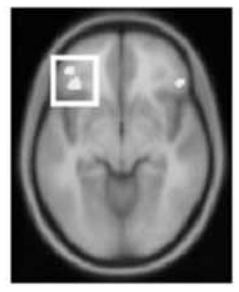

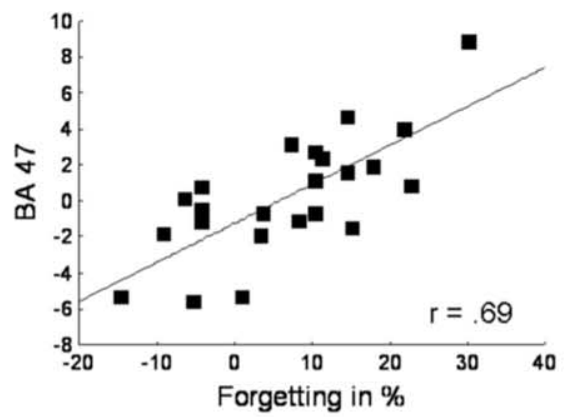
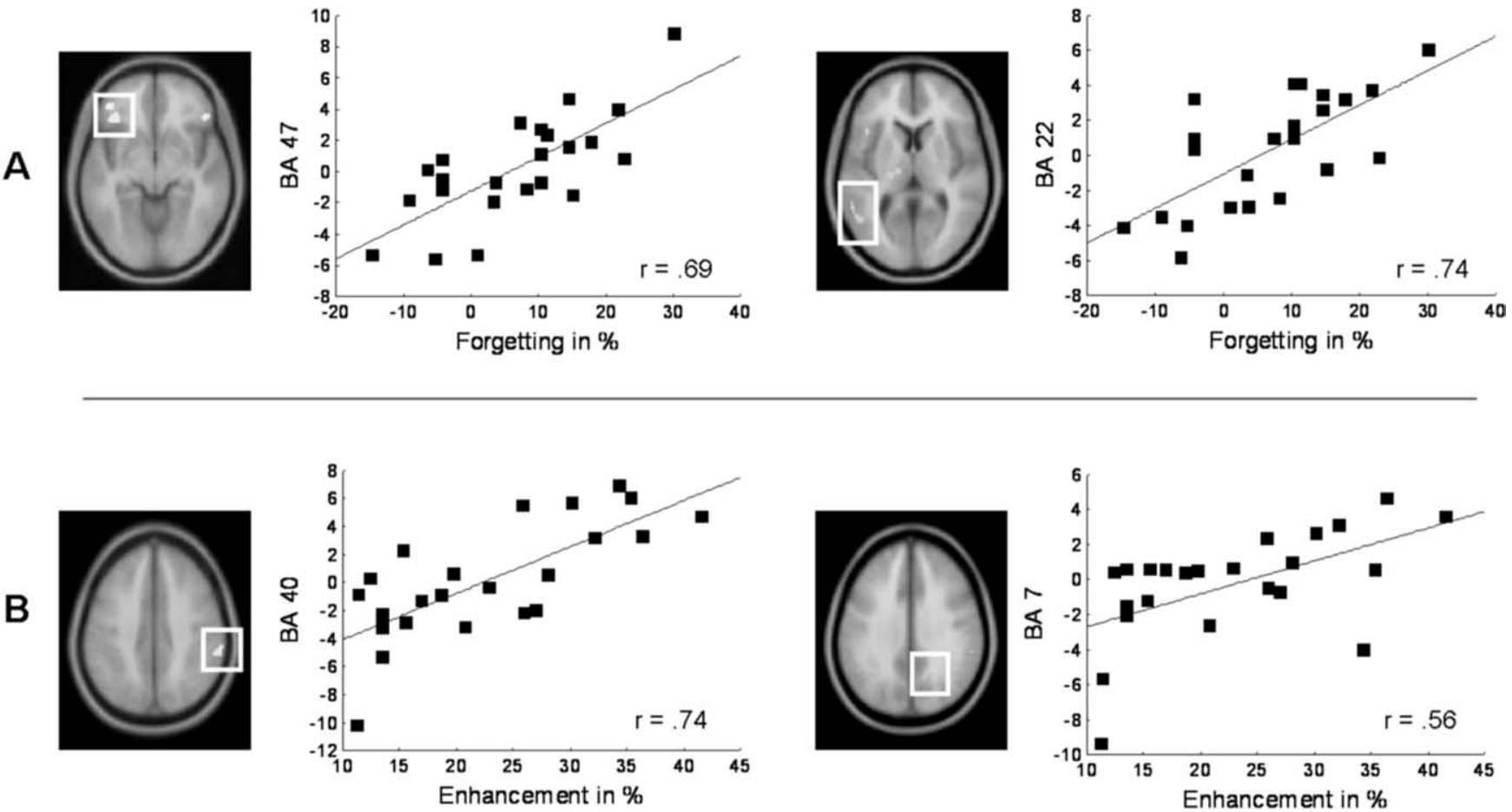

Figure 5. Brain-behavior correlations. $\boldsymbol{A}$, Regions where retrieval-induced forgetting (Forgetting in \%) was significantly correlated with differences in hemodynamic activity ( $\beta$ estimates) during the final cued recall test between $\mathrm{P}^{-}$and $\mathrm{P}^{+}$items. The highest correlations were found in the left inferior frontal gyrus (BA 47), and in the left superior temporal gyrus (BA 22). $\boldsymbol{B}$, Regions where retrieval-induced enhancement (Enhancement in $\%)$ was significantly $\left(p<0.001\right.$, uncorrected) correlated with differences in final recall activity $\left(\beta\right.$ estimates) between $P^{-}$and $P^{+}$items. The highest correlations were found in the right precuneus (BA7) and inferior parietal lobule (BA 40). All maps are thresholded with $p<0.001$ (uncorrected), and overlaid onto a standard anatomical T1 volume in SPM2 space.

strongest impairment-related pattern in support of the inhibitory account of retrieval-induced forgetting.

Interestingly, we found impairment-specific effects in areas that support controlled retrieval from semantic memory (Gold et al., 2006; Badre and Wagner, 2007). Although the standard retrieval practice paradigm used here strongly relies on semantic relations between memory representations (Bäuml, 2002), retrieval-induced forgetting has also been shown in purely episodic contexts (Ciranni and Shimamura, 1999). Future research might investigate whether the neural pattern observed here generalizes to such purely episodic interference.

\section{Conclusions}

The brain requires cognitive control processes that keep unwanted interfering information in memory from coming to mind (e.g., Bäuml, 2008; Levy and Anderson, 2002; RichardsonKlavehn and Bjork, 2002; Richardson-Klavehn et al., 2009). The present fMRI study revealed the neural substrates of the impaired retrieval of such interfering information in memory, at the time the impairment is observed. Impairment-specific hemodynamic changes occurred in ventrolateral prefrontal areas that are critical for the controlled retrieval of weak semantic representations. Our findings strengthen the view that retrieval-induced forgetting operates via inhibition that lowers the availability of memory representations.

\section{References}

Anderson MC (2003) Rethinking interference theory: executive control and the mechanisms of forgetting. J Mem Lang 49:415-445.

Anderson MC, Bell T (2001) Forgetting our facts: the role of inhibitory processes in the loss of propositional knowledge. J Exp Psychol Gen 130:544-570.

Anderson MC, Neely JH (1996) Interference and inhibition in memory re- trieval. In: Memory. Handbook of perception and cognition, Ed 2 (Bjork EL, Bjork RA, eds), pp 237-313. San Diego: Academic.

Anderson MC, Spellman BA (1995) On the status of inhibitory mechanisms in cognition: memory retrieval as a model case. Psychol Rev 102:68-100.

Anderson MC, Bjork RA, Bjork EL (1994) Remembering can cause forgetting: retrieval dynamics in long-term memory. J Exp Psychol Learn Mem Cogn 20:1063-1087.

Anderson MC, Green C, McCulloch KC (2000) Similarity and inhibition in long-term memory: evidence for a two-factor model. J Exp Psychol Learn Mem Cogn 26:1141-1159.

Badre D, Wagner AD (2007) Left prefrontal cortex and the cognitive control of memory. Neuropsychologia 45:2883-2901.

Badre D, Poldrack RA, Paré-Blagoev EJ, Insler RZ, Wagner AD (2005) Dissociable controlled retrieval and generalized selection mechanisms in ventrolateral prefrontal cortex. Neuron 47:907-918.

Battig WF, Montague WE (1969) Category norms for verbal items in 56 categories: a replication and extension of the Connecticut category norms. J Exp Psychol Monographs 80:1-46.

Bäuml K-H (1998) Strong items get suppressed, weak items do not: the role of item strength in output interference. Psychon Bull Rev 5:459-463.

Bäuml K-H (2002) Semantic generation can cause episodic forgetting. Psychol Sci 13:357-361.

Bäuml K-H (2008) Inhibitory processes. In: Learning and memory: a comprehensive reference, $\mathrm{Vol} 2$, Cognitive psychology of memory (Roediger HL 3rd, ed), pp 195-220. Oxford: Elsevier.

Bäuml K-H, Zellner M, Vilimek R (2005) When remembering causes forgetting: retrieval-induced forgetting as recovery failure. J Exp Psychol Learn Mem Cogn 31:1221-1234.

Ciranni MA, Shimamura AP (1999) Retrieval-induced forgetting in episodic memory. J Exp Psychol Learn Mem Cogn 25:1403-1414.

Friston KJ, Holmes AP, Worsley KJ, Poline JP, Frith CD, Frackowiak RSJ (1994) Statistical parametric maps in functional imaging: a general linear approach. Hum Brain Mapp 2:189-210.

Gold BT, Balota DA, Jones SJ, Powell DK, Smith CD, Andersen AH (2006) Dissociation of automatic and strategic lexical-semantics: functional 
magnetic resonance imaging evidence for differing roles of multiple frontotemporal regions. J Neurosci 26:6523-6532.

Gómez-Ariza CJ, Lechuga MT, Pelegrina S, Bajo MT (2005) Retrievalinduced forgetting in recall and recognition of thematically related and unrelated sentences. Mem Cognit 33:1431-14441.

Henson RN, Rugg MD (2003) Neural response suppression, hemodynamic repetition effects, and behavioural priming. Neuropsychologia 41:263-270.

Hicks JL, Starns JJ (2004) Retrieval-induced forgetting occurs in tests of item recognition. Psychon Bull Rev 11:125-130.

Johansson M, Aslan A, Bäuml K-H, Gäbel A, Mecklinger A (2007) When remembering causes forgetting: electrophysiological correlates of retrieval-induced forgetting. Cereb Cortex 17:1335-1341.

Johnson SK, Anderson MC (2004) The role of inhibitory control in forgetting semantic knowledge. Psychol Sci 15:448-453.

Kuhl BA, Dudukovic NM, Kahn I, Wagner AD (2007) Decreased demands on cognitive control reveal the neural processing benefits of forgetting. Nat Neurosci 10:908-914.

Kuhl BA, Kahn I, Dudukovic NM, Wagner AD (2008) Overcoming suppression in order to remember: contributions from anterior cingulate and ventrolateral prefrontal cortex. Cogn Affect Behav Neurosci 8:211-221.

Levy BJ, Anderson MC (2002) Inhibitory processes and the control of memory retrieval. Trends Cogn Sci 6:299-305.

Levy BJ, McVeigh ND, Marful A, Anderson MC (2007) Inhibiting your native language: the role of retrieval-induced forgetting during secondlanguage acquisition. Psychol Sci 18:29-34.

Richardson-Klavehn A, Bjork RA (2002) Memory, long-term. In: Encyclopedia of cognitive science, Vol 2 (Nadel L, ed), pp 1096-1105. London: Nature Publishing Group.

Richardson-Klavehn A, Magno E, Markopoulos G, Sweeney-Reed CM, Wimber M (2009) On the intimate relationship between neurobiology and function in the theoretical analysis of human learning and memory. In: Neuroimaging and psychological theories of human memory (Rösler F, Ranganath C, Röder B, Kluwe RH, eds). New York: Oxford UP.
Rundus D (1973) Negative effects of using list items as recall cues. J Verb Learn Verb Behav 12:43-50.

Schacter DL, Wig GS, Stevens WD (2007) Reductions in cortical activity during priming. Curr Opin Neurobiol 17:171-176.

Scheithe K, Bäuml K-H (1995) Deutschsprachige Normen für Vertreter von 48 Kategorien [German normative data for representatives of 48 categories]. Sprache und Kognition 14:39-43.

Schott BH, Henson RN, Richardson-Klavehn A, Becker C, Thoma V, Heinze H-J, Düzel E (2005) Redefining implicit and explicit memory: the functional neuroanatomy of priming, remembering, and control of retrieval. Proc Natl Acad Sci U S A 102:1257-1262.

Spitzer B, Bäuml K-H (2007) Retrieval-induced forgetting in item recognition: evidence for a reduction in general memory strength. J Exp Psychol Learn Mem Cogn 33:863-875.

Spitzer B, Hanslmayr S, Opitz B, Mecklinger A, Bäuml K-H (2008) Oscillatory correlates of retrieval-induced forgetting in recognition memory. J Cogn Neurosci. Advance online publication. Retrieved November 10, 2008. doi:10.1162/jocn.2009.21072

Veling H, van Knippenberg A (2004) Remembering can cause inhibition: retrieval-induced inhibition as cue independent process. J Exp Psychol Learn Mem Cogn 30:315-318.

Wagner AD, Shannon BJ, Kahn I, Buckner RL (2005) Parietal lobe contributions to episodic memory retrieval. Trends Cogn Sci 9:445-453.

Wheeler ME, Buckner RL (2003) Functional dissociation among components of remembering: control, perceived oldness, and content. J Neurosci 23:3869-3880.

Williams CC, Zacks RT (2001) Is retrieval-induced forgetting an inhibitory process? Am J Psychol 114:329-354.

Williams EJ (1959) The comparison of regression variables. R Stat Soc (Ser A) 46:63-76.

Wimber M, Rutschmann RM, Greenlee MW, Bäuml K-H (2008) Retrieval from episodic memory: neural mechanisms of interference resolution. J Cogn Neurosci. Advance online publication. Retrieved November 10, 2008. doi:10.1162/jocn.2009.21043 